\title{
Accommodation and Related Risk Factors Associated with Myopia Progression and Their Interaction with Treatment in COMET Children
}

\author{
Jane E. Gwiazda, ${ }^{1}$ Leslie Hyman, ${ }^{2}$ Thomas T. Norton, ${ }^{3}$ Mobamed E. M. Hussein, ${ }^{2}$ \\ Wendy Marsh-Tootle, ${ }^{3}$ Ruth Manny, ${ }^{4}$ Ying Wang, ${ }^{2}$ Donald Everett, ${ }^{5}$ and \\ the COMET Group 6 ,7
}

Purpose. To examine baseline measurements of accommodative lag, phoria, reading distance, amount of near work, and level of myopia as risk factors for progression of myopia and their interaction with treatment over 3 years, in children enrolled in the Correction of Myopia Evaluation Trial (COMET).

Methods. COMET enrolled 469 ethnically diverse children (ages, 6-11 years) with myopia between -1.25 and $-4.50 \mathrm{D}$. They were randomly assigned to either progressive addition lenses (PALs) with a +2.00 addition $(n=235)$ or single vision lenses (SVLs; $n=234$ ), the conventional spectacle treatment, and were observed for 3 years. The primary outcome measure was progression of myopia by autorefraction after cycloplegia with 2 drops of $1 \%$ tropicamide. Other measurements included accommodative response (by an open field of view autorefractor), phoria (by cover test), reading distance, and hours of near work. Independent and interaction analyses were based on the mean of the two eyes. Results were adjusted for important covariates with multiple linear regression.

RESULTS. Children with larger accommodative lags $(>0.43 \mathrm{D}$ for a $33 \mathrm{~cm}$ target) wearing SVLs had the most progression at 3 years. PALs were effective in slowing progression in these children, with statistically significant 3-year treatment effects (mean $\pm \mathrm{SE}$ ) for those with larger lags in combination with near esophoria $(\mathrm{PAL}-\mathrm{SVL}$ progression $=-1.08 \mathrm{D}-[-1.72$ $\mathrm{D}]=0.64 \pm 0.21 \mathrm{D})$, shorter reading distances $(0.44 \pm 0.20$ $\mathrm{D})$, or lower baseline myopia $(0.48 \pm 0.15 \mathrm{D})$. The 3 -year treatment effect for larger lags in combination with more hours of near work was $0.42 \pm 0.26 \mathrm{D}$, which did not reach statistical significance. Statistically significant treatment effects were observed in these four groups at 1 year and became larger from 1 to 3 years.

From the ${ }^{1}$ New England College of Optometry, Boston, Massachusetts; ${ }^{2}$ Stony Brook University School of Medicine, Stony Brook, New York; ${ }^{3}$ University of Alabama at Birmingham School of Optometry, Birmingham, Alabama; ${ }^{4}$ University of Houston College of Optometry, Houston, Texas; ${ }^{5}$ National Eye Institute, National Institute of Health, Bethesda, Maryland; and ' Pennsylvania College of Optometry, Philadelphia, Pennsylvania.

${ }^{7}$ COMET group members are listed in the appendix.

Supported by National Eye Institute Grants EY11805, EY11756, EY11754, EY11740, EY11752, and EY11755 and by Essilor of America, Marchon Eyewear, Marco Technologies, and Welch Allyn.

Submitted for publication December 2, 2003; revised February 6, 2004; accepted March 7, 2004.

Disclosure: J.E. Gwiazda, None; L. Hyman, None; T.T. Norton, None; M.E.M. Hussein, None; W. Marsh-Tootle, None; R. Manny, None; Y. Wang, None; D. Everett, None

The publication costs of this article were defrayed in part by page charge payment. This article must therefore be marked "advertisement" in accordance with 18 U.S.C. $\$ 1734$ solely to indicate this fact

Corresponding author: Jane E. Gwiazda, The New England College of Optometry, 424 Beacon Street, Boston, MA 02115;

gwiazdaj@ne-optometry.edu.
Conclusions. The results support the COMET rationale (i.e., a role for retinal defocus in myopia progression). In clinical practice in the United States children with large lags of accommodation and near esophoria often are prescribed PALs or bifocals to improve visual performance. Results of this study suggest that such children, if myopic, may have an additional benefit of slowed progression of myopia. (Invest Ophthalmol Vis Sci. 2004;45:2143-2151) DOI:10.1167/iovs.03-1306

$\mathrm{T}$ he Correction of Myopia Evaluation Trial (COMET) was a prospective, randomized clinical trial that evaluated the effect of progressive addition lenses (PALs) compared with single vision lenses (SVLs) on the progression of juvenile-onset myopia. ${ }^{1-3}$ Four hundred sixty-nine ethnically diverse $6-$ to 11-year-old children with baseline spherical equivalent myopia between -1.25 and $-4.50 \mathrm{D}$ were randomized to either SVLs, the conventional correction for myopia, or PALs with a +2.0 addition. The rationale for the trial was that PALs might slow myopia progression by reducing the amount of retinal defocus experienced by the children. Additional support for the trial was provided by a report that PALs significantly slowed the progression of myopia by approximately $0.50 \mathrm{D}$ after 2 years in 80 Chinese children. ${ }^{4}$ More recently, smaller treatment effects have been reported in two other studies of PALs and SVLs in Chinese children. ${ }^{5,6}$

The primary outcome of COMET was a statistically significant $(P=0.004)$ but clinically inconsequential adjusted difference in 3-year myopia progression of $0.20 \pm 0.08 \mathrm{D}$. Myopia in PAL wearers increased by $-1.28 \pm 0.06 \mathrm{D}$ and by $-1.48 \pm$ $0.06 \mathrm{D}$ in SVL wearers. In addition to measurement of myopia by cycloplegic autorefraction, COMET provided careful measurement of other factors, including accommodation and phorias, collected by certified examiners. These data allow for a comprehensive evaluation of risk factors associated with increased progression of myopia in this group of children. Risk factors independently related to the progression of myopia included age, gender, and ethnicity, which are presented in another paper (Hyman L. IOVS 2004; 45:ARVO E-Abstract 2734).

This article presents baseline accommodation and related risk factors (phoria, reading distance, and reported amount of near work), for the progression of myopia over 3 years in COMET children. Baseline myopia is also evaluated, since, like accommodation, it has been found to interact with treatment. These interactions were anticipated based on previous research in children and animals, summarized later, which provided part of the rationale for COMET. As modeled quantitatively by Flitcroft, ${ }^{7}$ the interaction of accommodative lag, near phoria, target distance, and time spent in near viewing could be important factors that determine whether eyes become myopic and whether the myopia progresses. In addition, this model suggests that myopic children with these factors could be helped with lenses such as bifocals or PALs. Another model that incorporates these factors is that of Hung and Ciuffreda. ${ }^{8,9}$ 


\section{Accommodation and Near Work}

When presented either with near targets or with minus-power lenses placed in front of the eyes, most individuals accommodate less than is needed to bring the target into focus. This underaccommodation is referred to as a lag of accommodation and is quantified by the difference between the accommodative stimulus and the measured accommodative response. A larger lag of accommodation in association with near work has been shown to be a factor in the development and progression of myopia. Larger accommodative lags in myopes are found with higher accommodative demands, ${ }^{10-12}$ myopia of more recent onset, ${ }^{11-13}$ and blur-driven accommodation induced by negative lenses. ${ }^{10,14,15}$ Larger lags also are associated with progression of myopia. ${ }^{14,15}$ The clinical measure of positive relative accommodation (PRA), which is accommodation stimulated by the addition of negative lenses to both eyes until blur is reported, is also reduced in myopes. ${ }^{16,17}$

A possible consequence of reduced accommodation to near targets, such as text during reading, is that myopes may experience extended periods of retinal defocus, which may lead to increased eye growth, especially vitreous chamber elongation, as has been demonstrated in animal models of myopia. ${ }^{18,19}$ Part of the rationale for COMET was based on providing clear vision with PALs to reduce defocus during near viewing and slow the progression of myopia.

Direct evidence of an association between near work and myopia has been difficult to obtain, in part because of challenges in measuring the amount of near work. Much of the existing evidence is provided by epidemiologic studies, ${ }^{20,21}$ and by prospective studies of the onset and progression of myopia in near work-intensive academic or occupational groups. ${ }^{22-24}$ Assessment of near work typically has been by questionnaire, with studies reporting a slight association between near work activities and myopia in children. Children in Singapore with more than $3 \mathrm{D}$ of myopia were reported to read significantly more than the median of two books per week and to engage in significantly more hours of daily near work than those with lower and no myopia. ${ }^{25}$ A greater amount of near work had a small independent contribution to myopia in a sample of U.S. children. ${ }^{26}$

In addition to high levels of near work, close viewing of text has been proposed as a risk factor for myopia, but limited data are available. Myopic children were found to hold text closer than emmetropic children, ${ }^{27}$ and progression of myopia was significantly greater in children with closer viewing distances. $^{28}$

\section{Phorias}

A shift in phoria toward esophoria is reported to occur while myopia is progressing, and faster progression occurs in children with near esophoria. ${ }^{16,29}$ Reduced accommodation has been found to be associated with near esophoria, ${ }^{30,31}$ leading to the suggestion that an esophoric child must relax accommodation to reduce accommodative convergence and thus maintain single binocular vision. The reduction in accommodation may produce hyperopic defocus during near work, which could lead to increased progression of myopia.

\section{Level of Refractive Error}

Limited data are available relating the amount of myopia at a given age to further progression, and often they are difficult to interpret because adjustments were not made for age of myopia onset, a known risk factor, and because the course of progression is not linear. Available data suggest that children with higher amounts of myopia at a point in time may show faster progression over a subsequent interval. Jensen reported 2-year progression of $0.64 \mathrm{D} / \mathrm{y}$ in children wearing SVLs and with baseline myopia of at least $2.75 \mathrm{D}$, compared with 2-year progression of $0.49 \mathrm{D}$ in children with less than $2.75 \mathrm{D}$, but the difference was not statistically significant. ${ }^{32}$ A retrospective study of clinical data from six practices found that faster progression of myopia was related to more myopia at the first examination, although age may have been a confounding variable. $^{33}$

In the present study, we examined baseline measurements of accommodative lag, phoria status, reading distance, reported near work, and amount of myopia as risk factors for progression of myopia over 3 years in COMET children.

\section{Methods}

Details of the study design and demographic characteristics of the study population have been published ${ }^{1-3}$ and are briefly summarized herein. Four clinical centers located at schools and colleges of optometry in Birmingham, Alabama; Boston, Massachusetts; Houston, Texas; and Philadelphia, Pennsylvania, enrolled 469 children between September 1997 and September 1998 and followed them for at least 3 years. Children enrolled in COMET met specific inclusion criteria, including the following: age 6 through 11 years inclusive at baseline, spherical equivalent between -4.50 and $-1.25 \mathrm{D}$ in each eye, astigmatism of $1.50 \mathrm{D}$ or less in either eye, anisometropia of $1.0 \mathrm{D}$ or less, birth weight of 1250 grams or more, visual acuity with distance correction of $0.20 \operatorname{logMAR}(20 / 32)$ or better, no strabismus by cover test at far $(4.0 \mathrm{~m})$ or near $(33 \mathrm{~cm})$ with the distance prescription, or at $33 \mathrm{~cm}$ wearing +2.0 over the distance prescription, and no prior wear of PALs or contact lenses.

Before the baseline examination, children and parents agreed that the children would accept random assignment of either SVLs or PALs, attend follow-up appointments semiannually for at least 3 years, and refrain from wearing contact lenses throughout the study. Children agreed to wear their COMET glasses during all waking hours. The COMET study and protocols conform to the tenets of the Declaration of Helsinki. The institutional review boards of each participating center approved the research protocols. Informed consent (parents) and assent (children) were obtained after verbal and written explanation of the nature and possible consequences of the study.

\section{Procedures}

Distance cycloplegic autorefraction was used to assess progression of myopia, the primary outcome measure. As with all data collection procedures, autorefraction was taken on both eyes by experienced optometrists who were trained and certified on study protocols. ${ }^{1-3}$ They also were masked to each child's lens assignment.

An autorefractor/autokeratometer (ARK 700A; Nidek, Gamagori, Japan) was used to take five consecutive, reliable readings both before and after cycloplegia. The cycloplegic agent was 2 drops of $1 \%$ tropicamide, administered 4 to 6 minutes apart, after corneal anesthesia was obtained with proparacaine for all but one child for whom benoxinate was used. The COMET protocol specified that cycloplegic autorefraction occur 30 minutes after administration of the second drop of tropicamide. Tropicamide (1\%) was found to be an effective cycloplegic agent in these myopic children, documented by residual accommodation measurements taken at baseline with an autorefractor (R-1; Canon, Lake Success, NY). ${ }^{34}$

Subjective refraction was completed before cycloplegia according to a standard protocol. ${ }^{1-3}$ At baseline all children received new glasses based on the distance refraction. At follow-up visits they received new glasses if the myopia correction, determined by subjective refraction, had increased by at least $0.50 \mathrm{D}$ spherical equivalent from the current prescription in at least one eye. Smaller prescription changes were made if clinically indicated.

Accommodation at near $(33 \mathrm{~cm})$ and far $(4.0 \mathrm{~m})$ for $20 / 100$ letter targets was measured monocularly using the Canon R-1 autorefractor. 
Because the Canon R-1, unlike most current autorefractors, has an open field of view to allow for presentation of real targets in free space, it has been the standard laboratory instrument for taking objective measurements of accommodation. The child wore a trial frame containing the best subjective spherical equivalent lens in front of the right eye, while viewing a target in free space in a dark room. The distant $(4.0 \mathrm{~m})$ target was a back-illuminated isolated row of 20/100 letters on an Early Treatment of Diabetic Retinopathy Study (ETDRS) chart. The contrast of the letters averaged $98 \%$ across the four participating centers. The near $(33 \mathrm{~cm})$ target was also a line of 20/100 letters of similar contrast. Instructions to the child were to keep the letters clear. Five accommodation readings were taken, excluding any measurements contaminated by blinks or extraneous eye movements.

Phoria at near $(33 \mathrm{~cm})$ and far $(4.0 \mathrm{~m})$ was measured using the cover test. The target was a 20/25 letter placed at $33 \mathrm{~cm}$ for the near measurements and at $4.0 \mathrm{~m}$ for the far measurements. A prism was used to neutralize the child's eye movements.

Three measurements of each child's normal reading distance for standardized age-appropriate text were taken at each visit, with the child wearing the latest prescription. Measurement was from the child's eye to the page of a book using a tape measure marked in inches. Values were converted to centimeters at the Coordinating Center. Near work diaries modified from a diary used by Jensen ${ }^{32}$ were completed for a week, soon after the study began. Children were asked to choose two typical school days and one typical weekend day on which to report activities. They checked off various activities performed at different time periods throughout the day. The data then were converted to diopter-hours, where a diopter-hour is a weighted measure that takes into account both the viewing distance and the duration of various activities. ${ }^{35}$

Myopia in children was corrected with either SVLs or PALs (Varilux Comfort lenses with a $+2.00 \mathrm{D}$ addition; Essilor International, St. Petersburg, FL); all lenses were polycarbonate. PALs were fitted with the top of the channel $4.0 \mathrm{~mm}$ above the pupil, allowing at least $11 \mathrm{~mm}$ for distance vision. The fitting protocol was designed to encourage the children to use the near addition portion of the lenses, because unlike presbyopic adults, for whom the glasses are typically prescribed, children can accommodate and thus are not dependent on the near addition for close work.

\section{Statistical Analyses}

Follow-up data were analyzed using an intent-to-treat principle according to the child's original lens assignment and the last known value of the outcome measures. For the seven children lost to follow-up and thus without data at the third annual visit, progression information from the latest follow-up visit was used.

Progression of myopia was defined as the change in spherical equivalent refractive error (SER) relative to baseline (a continuous measure). A summary measure of SER was calculated for each of the five autorefraction measurements per eye, and the mean of the five SER measures was then computed. The primary analysis for progression of myopia in COMET was child based, using the average of the two eyes to evaluate the magnitude of change in SER between follow-up and baseline (Pearson correlation coefficient between the two eyes at 3 years $=0.90$, and the mean interocular difference $<0.25 \mathrm{D}$ with a $95 \%$ confidence interval $[\mathrm{CI}]$ that includes 0 ).

The analytic strategy for this article used two main approaches. Univariate analyses were used to estimate the magnitude and variability of the risk posed by the levels of each covariate. Statistical modeling techniques were used to: (1) determine whether the covariate-specific risk interacted with the levels of the other covariates, (2) adjust the estimated covariate-specific risk for the effects of other predictors of myopia progression, and (3) test the statistical significance of these effects.

Univariate analyses were conducted using either the two-sample $t$-test if normality assumptions were preserved or the Wilcoxon sum rank test. These analyses were pursued partially to guide the selection of variables to be included in subsequent multivariate (modeling) analyses. Initially, each variable was evaluated to determine the magnitude of the difference in 3-year progression among its different levels.

The multivariate analyses used multiple linear regression to adjust for the potentially most prognostic covariates: age, gender, ethnicity, baseline refractive error, accommodative lag, and phoria, chosen because of their known relationship to the progression of myopia. Criteria for model building were based on first exploring and identifying an independent model and then hierarchically building an interaction model by sequential testing of each interaction term to be added to the model. These criteria were guided by the log likelihood principle. ${ }^{36}$ Testing for interaction was conducted using specific macros in the statistical analysis software (SAS Institute Inc., SAS, Cary, NC) to obtain estimates of a possible differential effect of PALs among categories of the selected covariates, and to test for the statistical significance of differences in progression of myopia between levels of one covariate cross-classified by levels of the other potentially interacting covariates. For example, to evaluate the interaction effect of treatment by accommodative lag, the difference between treatment groups for each level of accommodative lag was evaluated and then tested to see whether these differences were the same.

Criteria for selecting multifactor interaction terms were based on the univariate findings and interpretability of the interaction terms. At most three-factor interaction terms were tested (e.g., treatment by accommodative lag by age, gender, ethnicity, baseline myopia, phoria, reading distance, or hours of near work). All reported analyses were additionally controlled for multiple comparisons (when applicable), and the resultant simultaneous 95\% CIs were computed, using the approach described by Little et al. ${ }^{37}$

\section{Covariates for Multivariate Analyses}

Demographic variables included age (6-9 and 10-11 years), gender, and ethnicity (parent-reported as African-American, Asian, Hispanic, mixed, and white). Accommodative lag was based on a median split by number of children (smaller lag $<0.43 \mathrm{D}$ and larger lag $\geq 0.43 \mathrm{D}$ ). Near point phoria $(33 \mathrm{~cm})$ was measured by the cover test and defined as orthophoria $(-1.0 \Delta$ to $+1.0 \Delta)$, esophoria $(\geq 2 \Delta)$, or exophoria $(\leq$ $-2 \Delta$ ). Reading distance was based on a median split, with a closer reading distance of less than $31.2 \mathrm{~cm}$ and a longer reading distance of $31.2 \mathrm{~cm}$ or more. Hours of near work were based on a median split, with more near work defined as more than 104.5 diopter-hours per week and less near work defined as 104.5 diopter-hours per week or less. ${ }^{35}$ Baseline refractive error was based on a median split, with less myopia $(\geq-2.25 \mathrm{D})$ and more myopia $(<-2.25 \mathrm{D})$.

\section{Results}

The 469 children enrolled in COMET had a mean age of 9.3 years at baseline, and $52 \%$ were girls. They were ethnically diverse, with 46\% white, 26\% African-American, 15\% Hispanic, 8\% Asian, and 5\% mixed. Mean baseline spherical equivalent myopia measured by cycloplegic autorefraction was $-2.40 \mathrm{D}$. As reported previously, baseline characteristics were balanced, with no statistically significant differences between treatment groups. Retention was excellent, with 462 (98.5\%) of 469 children completing 3-year visits. Two children changed lens assignments, both from SVLs to PALs due to binocular vision problems. Of 2939 possible study visits from the children with 3 -year visits, only 10 were missed. Self-reported adherence to wearing glasses was excellent, with at least $93 \%$ of the PAL group and $96 \%$ of the SVL group reporting wearing their glasses most or all the time.

\section{Baseline Data}

Table 1 presents the amount of myopia at baseline in COMET PAL and SVL children by lag of accommodation and myopia. It 
TABLE 1. Baseline Myopia* by Accommodative Lag and Level of Myopia

\begin{tabular}{lcccc}
\hline & \multicolumn{4}{c}{ Baseline Myopia (D) } \\
\cline { 2 - 5 } \multicolumn{1}{c}{ Baseline Factors } & $\boldsymbol{n}$ & PAL & $\boldsymbol{n}$ & SVL \\
\hline Lag of accommodation (D) $\dagger$ & & & & \\
Smaller lag $(<0.43 \ddagger)$ & 120 & $-2.30 \pm 0.06$ & 115 & $-2.24 \pm 0.07$ \\
Larger lag $(\geq 0.43)$ & 114 & $-2.53 \pm 0.07$ & 119 & $-2.50 \pm 0.08$ \\
$P \Phi$ & & 0.03 & & 0.03 \\
Level of myopia (D) & & & & \\
$\quad$ Less myopia $(\geq-2.25 \ddagger)$ & 108 & $-1.75 \pm 0.03$ & 127 & $-1.72 \pm 0.03$ \\
More myopia $(<-2.25)$ & 126 & $-2.98 \pm 0.05$ & 107 & $-3.14 \pm 0.06$ \\
$P \Phi$ & & $<0.0001$ & & $<0.0001$ \\
Total & 234 & $-2.40 \pm 0.75$ & 234 & $-2.37 \pm 0.84$ \\
& & & &
\end{tabular}

Data are the mean $\pm \mathrm{SE}$.

* Based on spherical equivalent cycloplegic autorefraction and the mean of the two eyes.

† One child in PAL had missing data.

\# Based on the median split of the baseline distribution.

$\S P$ is based on the Wilcoxon rank sum test (due to non-normality) for testing a zero difference.

was interesting that, even at baseline, the children with larger accommodative lags had significantly more myopia than children with smaller lags, whether assigned to PALs $(P=0.03)$ or SVLs $(P=0.03)$. This finding was further confirmed by multivariate analyses controlling for the effects of age, gender, and ethnicity. The mean amount of myopia at baseline was, by definition, significantly greater in the high myopia group than in the low myopia group.

\section{Variables Interacting with Treatment}

Lag of accommodation and amount of myopia were the two variables found to interact significantly with treatment in COMET children. ${ }^{1}$ As shown in Table 2 , the treatment effect was significantly larger in children with larger lags than in those with smaller lags $(0.33 \mathrm{D}$ vs. $0.07 \mathrm{D})$. Adjusted 3-year progression of myopia in PAL children was similar regardless of the level of accommodative lag (adjusted difference, $-0.01 \mathrm{D}$ ). In the SVL group, the difference in progression between lag groups $(0.24 \mathrm{D})$ was significantly larger than for the PAL group. This difference resulted from greater progression in SVL children with larger versus smaller lags $(1.60 \mathrm{D}$ vs. $1.36 \mathrm{D})$. Over- all, the interaction effect between lag and treatment on myopia progression was $0.26 \mathrm{D}(P<0.05)$. This value is the estimated average increase in progression for larger lags in SVL compared with PAL children. Equivalently, it is also the estimated benefit of PALs for children with larger compared with smaller lags.

For baseline amount of myopia, Table 2 shows that the treatment effect was significantly larger in children with lower versus higher baseline myopia $(0.30 \mathrm{D}$ vs. $0.10 \mathrm{D})$. The 3 -year adjusted progression was smaller in the PAL group with less compared with more myopia, but was similar in the two SVL groups. Overall, the interaction effect on progression between amount of myopia and treatment was $0.20 \mathrm{D}(P<0.05)$. This value is the estimated average increase in progression in children with less myopia in the SVL compared with the PAL group. Equivalently, it is also the estimated benefit of PALs for children with less compared with more myopia.

\section{Three-Year Myopia Progression by Lag of Accommodation and Treatment}

Additional interaction analyses were conducted to examine 3-year myopia progression stratified by lag of accommodation and treatment group. Figure 1 shows the unadjusted treatment effect in children with larger and smaller accommodative lags for phoria (Fig. 1A), reading distance (Fig. 1B), baseline myopia (Fig. 1C), and near work duration (Fig. 1D). In Figure 1A, the largest treatment effect $(0.77 \mathrm{D})$ was observed in children with larger accommodative lags and near esophoria. This difference is large, because children with larger lags and near esophoria wearing PALs had the least progression $(0.98 \mathrm{D})$, whereas the greatest progression $(1.75 \mathrm{D})$ occurred in children with larger lags and near esophoria wearing SVLs. Children with orthophoria, regardless of the level of lag, showed an intermediate treatment effect, whereas exophoric children showed small differences between the PAL and SVL groups in the opposite direction. For reading distance, a greater treatment effect was observed in children with larger lags, especially with closer reading distances (Fig 1B). As shown in Fig 1C, children with less baseline myopia and larger lags of accommodation had a larger treatment effect than did children with less myopia/ smaller lags or those with more myopia. Considering near work, children who performed more hours of near work per week ( $>104.5$ diopter-hours) and had larger lags of accommodation showed a greater treatment effect than children with

TABle 2. Adjusted* Myopia Progression and Treatment Effect at 3 Years by Level of Baseline Accommodative Lag and Myopia

Three-Year Progression (D)

\begin{tabular}{|c|c|c|c|c|c|c|}
\hline \multirow[b]{2}{*}{ Baseline Factors } & \multicolumn{2}{|c|}{ PAL } & \multicolumn{2}{|c|}{ SVL } & \multicolumn{2}{|c|}{$\begin{array}{l}\text { Treatment Effect } \\
\text { (PAL - SVL) }\end{array}$} \\
\hline & $n$ & Mean $\pm \mathrm{SE}$ & $n$ & Mean \pm SE & Mean \pm SE & (95\% CI) \\
\hline \multicolumn{7}{|l|}{ Accommodative lag (D) $\dagger$} \\
\hline Smaller lag $(<0.43)$ & 120 & $-1.28 \pm 0.08$ & 115 & $-1.36 \pm 0.08$ & $0.07 \pm 0.11$ & $(-0.18,0.33)$ \\
\hline Larger lag $(\geq 0.43)$ & 114 & $-1.27 \pm 0.08$ & 119 & $-1.60 \pm 0.08$ & $0.33 \pm 0.11 \neq$ & $(0.07,0.58)$ \\
\hline $\begin{array}{l}\text { Adjusted difference } \pm \mathrm{SE} \\
(95 \% \mathrm{CI})\end{array}$ & & $\begin{array}{l}-0.01 \pm 0.10 \\
(-0.27,0.25)\end{array}$ & & $\begin{array}{r}0.24 \pm 0.10 \\
(-0.02,0.50)\end{array}$ & & \\
\hline \multicolumn{7}{|l|}{ Myopia (D)† } \\
\hline More myopia $(<-2.25)$ & 126 & $-1.38 \pm 0.08$ & 107 & $-1.48 \pm 0.08$ & $0.10 \pm 0.11$ & $(-0.15,0.36)$ \\
\hline Less myopia $(\geq-2.25)$ & 108 & $-1.17 \pm 0.08$ & 127 & $-1.47 \pm 0.08$ & $0.30 \pm 0.11 \neq$ & $(0.04,0.55)$ \\
\hline Adjusted difference $\pm \mathrm{SE}$ & & $-0.21 \pm 0.10$ & & $-0.01 \pm 0.10$ & & \\
\hline$(95 \% \mathrm{CI})$ & & $(-0.47,0.04)$ & & $(-0.27,0.23)$ & & \\
\hline
\end{tabular}

*Adjusted for age, gender, ethnicity, baseline myopia, phoria, accommodative lag, and treatment $(n=468)$.

† Statistically significant interaction with treatment $(P<0.05)$.

‡ Statistically significant $(P<0.05)$. 

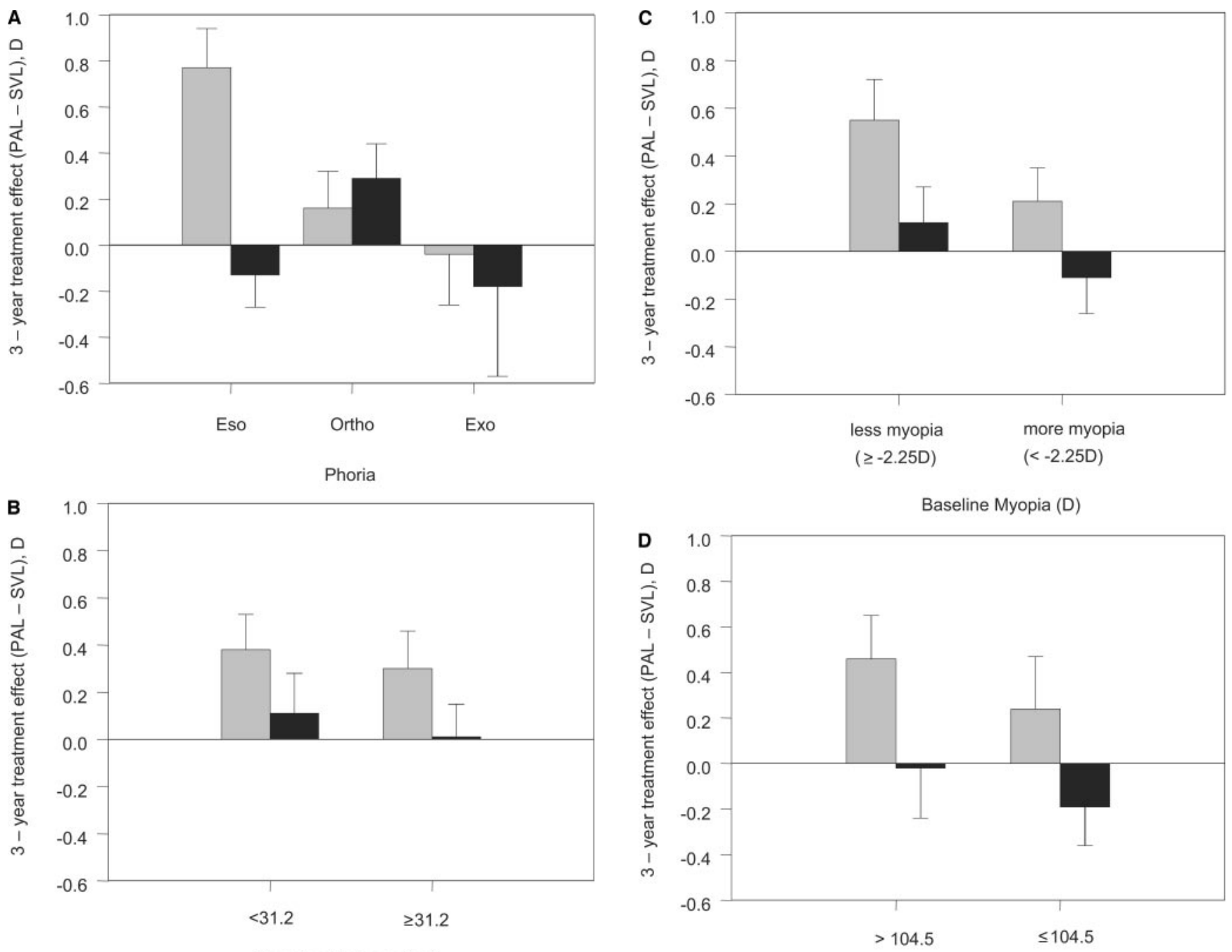

Reading Distance $(\mathrm{cm})$

\section{Near Work (diopter hours / week)}

FigURE 1. Unadjusted treatment effects (PAL - SVL) in children with larger $\square, \geq 0.43 \mathrm{D}$ ) and smaller ( $\square,<0.43 \mathrm{D}$ ) accommodative lags for phoria (A), reading distance (B), baseline myopia (C), and near work duration (D).

more near work/smaller lags and than those with less near work (Fig 1D).

Table 3 shows adjusted 3-year myopia progression by level of accommodative lag for both the PAL and SVL groups for the covariates described earlier. The adjusted results are similar to the unadjusted shown in Figure 1. For all factors evaluated except exophoria, the greatest progression occurred in children with larger lags of accommodation who were wearing SVLs. In addition, statistically significant treatment effects were found within the baseline factors in children with larger accommodative lags, whereas no significant treatment effects were found in children with smaller lags.

With respect to phorias, the least progression $(1.08 \mathrm{D})$ occurred in children with large accommodative lags and near esophoria wearing PALs and the greatest progression (1.72 D) in children with the same characteristics wearing SVLs, resulting in a statistically significant adjusted treatment effect of 0.64 D. Treatment effects were smaller in orthophores and similar for both large and small lags, whereas for exophores they were negligible. The correlation between accommodative lag and near phoria was $-0.16(P<0.001)$.
For reading distance, the greatest progression occurred in children with larger accommodative lags and closer reading distances wearing SVLs $(1.68 \mathrm{D})$, which, when compared with the progression found in children with the same characteristics wearing PALs $(1.24 \mathrm{D})$ resulted in a statistically significant treatment effect of $0.44 \mathrm{D}$. The correlation between accommodative lag and reading distance was -0.08 ( $P=0.09)$.

Considering baseline level of myopia, the greatest 3-year progression of myopia occurred in children with a larger accommodative lag wearing SVLs, regardless of the level of myopia. The smallest progression occurred in PAL children with a larger lag and low baseline myopia $(1.11 \mathrm{D})$, which when compared with the progression in SVL children with the same characteristics $(1.59 \mathrm{D})$ resulted in a statistically significant treatment effect of $0.48 \mathrm{D}$.

For near work, Table 3 shows that children wearing SVLs with a larger accommodative lag had the most progression. Children in PALs with a larger lag who performed more near work than the median of 104.5 diopter-hours per week had myopia progression of $1.14 \mathrm{D}$, resulting in a treatment effect of 
Table 3. Adjusted Myopia Progression and Treatment Effect in Diopters at 3 Years, Stratified by Level of Baseline Accommodative Lag and Lens Type for Baseline Factors of Phoria, Reading Distance, Level of Myopia, and Near Work

Three-Year Progression (D)

\begin{tabular}{|c|c|c|c|c|c|c|c|c|c|c|}
\hline \multirow[b]{3}{*}{ Baseline Factors } & \multicolumn{5}{|c|}{ Larger Lag $(\geq 0.43 \mathrm{D})$} & \multicolumn{5}{|c|}{ Smaller Lag $(<0.43 \mathrm{D})$} \\
\hline & \multicolumn{2}{|r|}{ PAL } & \multicolumn{2}{|c|}{ SVL } & \multirow[b]{2}{*}{$\begin{array}{c}\text { Diff. PAL - SVL } \\
(95 \% \mathrm{CI})\end{array}$} & \multicolumn{2}{|c|}{ PAL } & \multicolumn{2}{|c|}{ SVL } & \multirow[b]{2}{*}{$\begin{array}{l}\text { Diff. PAL - SVL } \\
\quad(95 \% \mathrm{CI})\end{array}$} \\
\hline & $n$ & Mean & $n$ & Mean & & $n$ & Mean & $n$ & Mean & \\
\hline \multicolumn{11}{|l|}{ Phoria $(\Delta)(\text { cover test })^{*}$} \\
\hline Eso $(\geq 2)$ & 42 & $-1.08 \S$ & 34 & -1.72 & $0.64 \|(0.08,1.19)$ & 55 & -1.24 & 55 & -1.15 & $-0.09(-0.55,0.37)$ \\
\hline Ortho $(-1-1)$ & 46 & -1.39 & 63 & -1.64 & $0.25(-0.22,0.72)$ & 46 & -1.14 & 45 & -1.49 & $0.35(-0.16,0.85)$ \\
\hline $\operatorname{Exo}(\leq-2)$ & 26 & -1.32 & 22 & -1.30 & $-0.02(-0.72,0.68)$ & 19 & -1.59 & 15 & -1.51 & $-0.08(-0.91,0.75)$ \\
\hline \multicolumn{11}{|l|}{ Reading distance $(\mathrm{cm}) \dagger$} \\
\hline$<31.2$ & 64 & -1.24 & 52 & -1.68 & $0.44 \|(0.01,0.86)$ & 64 & -1.34 & 44 & -1.48 & $0.14(-0.30,0.59)$ \\
\hline$\geq 31.2$ & 49 & -1.33 & 62 & -1.54 & $0.21(-0.21,0.65)$ & 56 & -1.23 & 69 & -1.30 & $0.07(-0.35,0.47)$ \\
\hline \multicolumn{11}{|l|}{ Level of myopia (D)* } \\
\hline Less myopia $(\geq-2.25)$ & 44 & -1.11 & 60 & -1.59 & $0.48 \|(0.02,0.93)$ & 64 & -1.19 & 67 & -1.35 & $0.16(-0.23,0.56)$ \\
\hline More myopia $(<-2.25)$ & 70 & -1.40 & 59 & -1.62 & $0.22(-0.18,0.61)$ & 56 & -1.38 & 48 & -1.34 & $-0.04(-0.49,0.40)$ \\
\hline \multicolumn{11}{|c|}{ Near work (diopter-hours/week) } \\
\hline$>104.5$ & 32 & -1.14 & 38 & -1.56 & $0.42(-0.12,0.96)$ & 26 & -1.22 & 31 & -1.33 & $0.11(-0.49,0.71)$ \\
\hline$\leq 104.5$ & 24 & -1.40 & 37 & -1.58 & $0.18(-0.42,0.77)$ & 38 & -1.34 & 31 & -1.12 & $-0.22(-0.77,0.33)$ \\
\hline
\end{tabular}

* Model includes age, gender, ethnicity, baseline myopia, phoria (cover test), lag, and treatment as covariates $(n=468)$.

† Model includes age, gender, ethnicity, baseline myopia, phoria (cover test), lag, treatment, and reading distance as covariates $(n=460)$.

‡ Model includes age, gender, ethnicity, baseline myopia, phoria (cover test), lag, treatment, and near work as covariates $(n=257)$.

$\$$ Standard errors ranged from 0.09 to 0.19

$\|$ Statistically significant $(P<0.05)$.

$0.42 \mathrm{D}$, which was not statistically significant, perhaps due to the smaller number of subjects with near work information.

\section{One-Year Myopia Progression by Lag of Accommodation and Treatment}

The statistically significant treatment effects found at 3 years for children with larger accommodative lags were also observed at 1 year, but the size of the treatment effect at 1 year was not yet at the level observed at 3 years, as shown in Table
4. Children with larger lags in combination with either near esophoria, closer reading distances, lower baseline myopia, or more hours of near work had the only statistically significant treatment effects at 1 year. The magnitudes of these 1-year effects were approximately $0.20 \mathrm{D}$ less than the treatment effects at 3 years. For example, at 1 year children with a larger accommodative lag and near esophoria had an adjusted treatment effect of $0.39 \mathrm{D}$, compared with an adjusted treatment effect of $0.64 \mathrm{D}$ at 3 years. The other groups of children with

TABLE 4. Adjusted Myopia Progression and Treatment Effect at 1 Year, Stratified by Level of Baseline Lag of Accommodation for Baseline Factors of Phoria, Reading Distance, Baseline Myopia and Near Work

One Year Progression

\begin{tabular}{|c|c|c|c|c|c|c|c|c|c|c|}
\hline \multirow[b]{3}{*}{ Baseline Factors } & \multicolumn{5}{|c|}{ Larger Lag $(\geq 0.43)$} & \multicolumn{5}{|c|}{ Smaller Lag $(<0.43)$} \\
\hline & \multicolumn{2}{|r|}{ PAL } & \multicolumn{2}{|c|}{ SVL } & \multirow{2}{*}{$\begin{array}{c}\text { Difference } \\
\text { PAL - SVL } \\
\text { (95\% CI) }\end{array}$} & \multicolumn{2}{|c|}{ PAL } & \multicolumn{2}{|c|}{ SVL } & \multirow{2}{*}{$\begin{array}{c}\text { Difference } \\
\text { PAL - SVL } \\
\text { (95\% CI) }\end{array}$} \\
\hline & $n$ & Mean & $n$ & Mean & & $n$ & Mean & $n$ & Mean & \\
\hline \multicolumn{11}{|l|}{ Phoria $(\Delta)^{*}$} \\
\hline Eso $(\geq 2)$ & 42 & $-0.37 \S$ & 34 & -0.76 & $0.39 \|(0.11,0.67)$ & 55 & -0.42 & 55 & -0.53 & $0.11(-0.12,0.34)$ \\
\hline Ortho $(-1-1)$ & 46 & -0.52 & 63 & -0.68 & $0.16(-0.08,0.39)$ & 46 & -0.38 & 45 & -0.60 & $0.22(-0.03,0.48)$ \\
\hline $\operatorname{Exo}(\leq-2)$ & 26 & -0.50 & 22 & -0.58 & $0.08(-0.27,0.43)$ & 19 & -0.61 & 15 & -0.64 & $0.03(-0.38,0.46)$ \\
\hline \multicolumn{11}{|l|}{ Reading distance $(\mathrm{cm}) \dagger$} \\
\hline$<31.2$ & 64 & -0.45 & 52 & -0.72 & $0.27 \|(0.06,0.49)$ & 64 & -0.43 & 44 & -0.64 & $0.21(-0.01,0.44)$ \\
\hline$\geq 31.2$ & 49 & -0.50 & 62 & -0.67 & $0.17(-0.04,0.39)$ & 56 & -0.47 & 69 & -0.56 & $0.09(-0.12,0.29)$ \\
\hline \multicolumn{11}{|l|}{ Baseline myopia (D)* } \\
\hline Less myopia $(\geq-2.25)$ & 44 & -0.38 & 60 & -0.65 & $0.28 \|(0.04,0.50)$ & 64 & -0.41 & 67 & -0.59 & $0.18(-0.02,0.38)$ \\
\hline More myopia $(<-2.25)$ & 70 & -0.54 & 59 & -0.73 & $0.19(-0.01,0.39)$ & 56 & -0.48 & 48 & -0.57 & $0.09(-0.13,0.32)$ \\
\hline \multicolumn{11}{|c|}{ Near work (diopter-hours/week) $\ddagger$} \\
\hline$>104.5$ & 32 & -0.48 & 38 & -0.76 & $0.27 \|(0.02,0.53)$ & 26 & -0.49 & 31 & -0.62 & $0.13(-0.16,0.42)$ \\
\hline$\leq 104.5$ & 24 & -0.50 & 37 & -0.65 & $0.15(-0.14,0.44)$ & 38 & -0.47 & 31 & -0.45 & $-0.02(-0.29,0.24)$ \\
\hline
\end{tabular}

* Model includes age, gender, ethnicity, baseline myopia, phoria (cover test), lag, and treatment as covariates $(n=468)$.

† Model includes age, gender, ethnicity, baseline myopia, phoria (cover test), lag, treatment, and reading distance as covariates $(n=460)$.

‡ Model includes age, gender, ethnicity, baseline myopia, phoria (cover test), lag, treatment, and near work as covariates $(n=257)$.

$\S$ Standard errors ranged from 0.05 to 0.10 .

$\|$ Statistically significant $(P<0.05)$. 
larger lags (i.e., those who also had orthophoria, longer reading distances, more baseline myopia, or fewer hours of near work) had similar, small treatment effects at both 1 and 3 years. Exophoric children with large lags had a negligible treatment effect at both 1 and 3 years. Most of the children with smaller accommodative lags at baseline showed little or no treatment effect at either 1 or 3 years. For all but one subgroup with smaller lags, the treatment effects decreased slightly between 1 and 3 years. Orthophoric children were an exception, with a treatment effect of $0.23 \mathrm{D}$ at 1 year and $0.35 \mathrm{D}$ at 3 years, but neither value was statistically significant.

\section{Baseline Myopia}

As with accommodative lag, additional interaction analyses were conducted to examine myopia progression by amount of baseline myopia and treatment group. However, unlike with the accommodation analyses, there was only one significant result, and that was for lag of accommodation. As reported previously, children with larger lags and less baseline myopia had a statistically significant 3-year treatment effect of $0.48 \mathrm{D}$.

\section{Discussion}

The current results provide additional support for a role of defocus in the progression of myopia in humans, consistent with the rationale for COMET. COMET children most at risk for increased progression of myopia over 3 years had, at baseline, a larger accommodative lag in combination with near esophoria and wore the conventional treatment for myopia (SVLs). PALs slowed progression in these children and also in those with larger accommodative lags in combination with a shorter reading distance, more hours of near work, or lower baseline myopia. Retinal defocus resulting from inaccurate accommodation in some myopic children when they are engaged in close work may be a stimulus for increased axial elongation and progression of myopia, as found in animal models. ${ }^{18,19}$ The benefits of PALs for these children are consistent with the view that retinal defocus is a stimulus to progression of myopia, and the treatment effect may be due to the ability of PALs to provide clear retinal images in these children.

Most myopic children are prescribed SVLs. ${ }^{38,39}$ For the COMET children wearing SVLs, the level of baseline myopia was not a significant risk factor, but a large accommodative lag, assessed objectively, was. Children with larger accommodative lags wearing SVLs showed the most progression, and children with larger lags wearing PALs showed reduced progression. The same pattern held across age, gender, and ethnicity, which is presented in more detail in a separate paper (Hyman L. IOVS 2004; 45:ARVO E-Abstract 2734). Briefly, younger children and girls with larger accommodative lags wearing SVLs showed the most progression. The number of subjects was large only for African-American and white children; both ethnic groups showed this same pattern of progression based on accommodative lag and lens type.

When phoria was combined with accommodation, progression at both 1 and 3 years was found to be smallest in children wearing PALs who had both a large accommodative lag and near esophoria and largest in children wearing SVLs with the same baseline characteristics. In part, because near esophoria has been strongly identified as a risk factor for progression of myopia, Fulk et al. ${ }^{40}$ conducted a clinical trial of 84 myopic children with near esophoria, randomized to either bifocals or SVLs. Overall, a 0.25-D treatment effect was found, similar in magnitude to other studies that did not limit enrollment by phoria status. ${ }^{4-6}$ Because accommodation was not measured in these other studies, it is not possible to evaluate the progression of myopia and the treatment effect by lag of accommodation. The COMET results, however, suggest that the diagnosis of near esophoria in the absence of information on accommodation may not be sufficient to determine the best spectacle lens treatment for myopic children.

Measurement of accommodation and phoria were combined in a retrospective reanalysis of patient records from three practices, ${ }^{41}$ using near, binocular, crossed-cylinder findings as a measure of lag of accommodation. Even though the number of subjects was very small, a pattern similar to the present results was found. Greater progression occurred in myopic children with large $(\geq 0.50 \mathrm{D})$ lags and near esophoria wearing SVLs $(0.55 \mathrm{D} / \mathrm{y})$ compared with those with the same characteristics wearing bifocals $(0.26 \mathrm{D} / \mathrm{y})$. The sample was too small to evaluate results for children with lags less than $0.50 \mathrm{D}$. Looking at lags alone, regardless of phoria status, progression for children with large lags was found to be significantly greater in the SVL group $(0.48 \mathrm{D} / \mathrm{y})$ than in the bifocal group $(0.25 \mathrm{D} / \mathrm{y})$. In patients with small lags, there was no difference in annual progression of myopia by lens type, approximately $0.40 \mathrm{D} / \mathrm{y}$ in each treatment group, a finding similar to that in the present study. These data suggest that careful measurement of accommodative lags is important for determining the best spectacle lens treatment for myopic children and presumably will be important for whatever type of treatment (e.g., contact lenses, drugs) is being considered.

To account for the observed association between larger accommodative lags and near esophoria in previous studies, it has been suggested that an esophoric child must relax accommodation to reduce accommodative convergence and thus maintain single binocular vision. ${ }^{30,31}$ In the present study the correlation between these two factors was found to be low, though significant, and so this explanation cannot be the whole story.

Baseline reading distance emerged as a risk factor for progression among children with close reading distances and larger accommodative lags wearing SVLs. Rosenfield et al. ${ }^{42}$ reported that children of ages (6.4-10.75 years) similar to those in the COMET group had a mean reading distance of 27.2 $\mathrm{cm}$, with no increase in reading distance over that age range. The median reading distance in the COMET group was 31.2 $\mathrm{cm}$, slightly greater than that in the earlier report. Because our near targets for measuring accommodation were at $33 \mathrm{~cm}$, this distance was most appropriate for the COMET children. Myopic children are reported to hold text and video games closer than emmetropic children. ${ }^{27}$ The COMET data showed that a near reading distance, especially in children with large lags wearing SVLs, led to faster progression, and that PALs slowed progression in this group of children.

The near work data present a plausible pattern of results, even with a reduced sample size and the inherent problems in assessing near work by questionnaire. A significant treatment effect at 1 year (which approached significance at 3 years) was found only for children with more hours of near work in combination with larger accommodative lags, suggesting that the lens intervention was more effective for those children who most likely experienced more blurred near vision. More sophisticated ways of assessing near work are being explored. ${ }^{43}$ In addition, temporal factors increasingly are found to play a role in animal models of myopia, ${ }^{44,45}$ suggesting that the timing of periods of near and distance viewing, and the type of spectacles worn or not worn, are likely to be of importance. Even with these limitations, the present data suggest that PALs may be beneficial for children with larger accommodative lags who perform extensive near work activities.

The literature provides limited data on the risk of myopia progression for different amounts of presenting myopia. COMET children wearing the conventional treatment, SVLs, had similar progression over 3 years, whether they had lower or higher baseline myopia. The children in PALs with lower baseline myopia had reduced progression compared with 
those in PALs who had higher myopia. Therefore, a statistically significant treatment effect was found only in children with lower baseline myopia. This result suggests that once the myopization process has started and passed some critical level, it may be more difficult to intervene with an optical treatment.

The present results suggest that the main 3-year treatment effect in COMET of $0.20 \mathrm{D}$, which was found at 1 year and sustained over the next 2 years of follow-up, ${ }^{1}$ is more representative of certain subsets of COMET children than others. Children with smaller baseline accommodative lags showed a limited treatment effect which was similar over 3 years, whereas those with larger lags at study onset, in combination with near esophoria, a closer reading distance, lower myopia, or more hours of near work, showed larger treatment effects that grew over 3 years. Increases in the size of the treatment effect between 1 and 3 years (approximately $0.20 \mathrm{D}$ ) were smaller than the effect that developed between baseline and 1 year. This result suggests that for children with larger lags at baseline, accommodation may have improved over the course of the study, leading to slowed efficacy of PALs. Future analyses of COMET data will show whether changes in the lag of accommodation over 3 years accounted for the present results.

Additional analyses also will show whether there are changes in the accommodative convergence-to-accommodation (AC/A) ratio over time and whether they are associated with progression of myopia. Elevated AC/A ratios have been linked to the development and progression of myopia. ${ }^{31,46}$ Higher AC/A ratios are found in myopic than in emmetropic children, because myopes have significantly reduced accommodation and slightly increased accommodative convergence. ${ }^{31}$ Accommodation and phoria measurements from COMET children at baseline and each year of follow-up will be used to calculate AC/A ratios, to be presented in a future paper.

In the United States, children who demonstrate near esophoria and poor accommodation, regardless of refractive error, often are prescribed PALs or bifocals to improve their visual performance. These same children, if myopic, may have an additional benefit of slowed progression of myopia. However, the present analyses, while hypothesis-driven, are based on subsets of the full COMET data set representing 469 children. Therefore, any clinical recommendations will have to await results from a follow-up study to establish the efficacy of PALs in slowing the progression of myopia in children with the risk profile reported in this article.

\section{References}

1. Gwiazda J, Hyman L, Hussein M, et al. A randomized clinical trial of progressive addition lenses versus single vision lenses on the progression of myopia in children. Invest Ophthalmol Vis Sci. 2003;44:1492-1500.

2. Hyman L, Gwiazda J, Marsh-Tootle WL, Norton TT, Hussein M, COMET Group. The Correction of Myopia Evaluation Trial (COMET): design and general baseline characteristics. Control Clin Trials. 2001;22:573-592.

3. Gwiazda J, Marsh-Tootle WL, Hyman L, Hussein M, Norton TT, COMET Study Group. Baseline refractive and ocular component measures of children enrolled in the correction of myopia evaluation trial (COMET). Invest Ophthalmol Vis Sci. 2002;43:314-321.

4. Leung JT, Brown B. Progression of myopia in Hong Kong Chinese schoolchildren is slowed by wearing progressive lenses. Optom Vis Sci. 1999;76:346-354.

5. Shih YF, Hsiao CK, Chen CJ, Chang CW, Hung PT, Lin LL. An intervention trial on efficacy of atropine and multi-focal glasses in controlling myopic progression. Acta Ophthalmol Scand. 2001; 79:233-236.

6. Edwards M, Li R, Lam C, Lew J, Yu B. The Hong Kong progressive lens myopia control study: study design and main findings. Invest Ophthalmol Vis Sci. 2002;43:2852-2858.
7. Flitcroft DI. A model of the contribution of oculomotor and optical factors to emmetropization and myopia. Vision Res. 1998;38: 2869-2879

8. Hung G, Ciuffreda K. Model of human refractive error development. Curr Eye Res. 1999;19:41-52.

9. Hung G, Ciuffreda K. Quantitative analysis of the effect of near lens addition on accommodation and myopigenesis. Curr Eye Res. 2000;20:293-312.

10. Gwiazda J, Thorn F, Bauer J, Held R. Myopic children show insufficient accommodative response to blur. Invest Ophthalmol Vis Sci. 1993;34:690-694.

11. McBrien NA, Millodot M. The effect of refractive error on the accommodative response gradient. Ophthalmic Physiol Opt. 1986; 6:145-149.

12. Rosenfield M, Gilmartin B. Disparity-induced accommodation in late-onset myopia. Ophthalmic Physiol Opt. 1988;8:353-355.

13. Bullimore MA, Gilmartin B, Royston JM. Steady-state accommodation and ocular biometry in late-onset myopia. Doc Ophthalmol. 1992;80:143-155.

14. Gwiazda J, Bauer J, Thorn F, Held R. A dynamic relationship between myopia and blur-driven accommodation in school-aged children. Vision Res. 1995;35:1299-1304.

15. Abbott ML, Schmid KL, Strang NC. Differences in the accommodation stimulus response curves of adult myopes and emmetropes. Ophthalmic Physiol Opt. 1998;18:13-20.

16. Goss DA. Clinical accommodation and heterophoria findings preceding juvenile onset of myopia. Optom Vis Sci. 1991;68:110-116.

17. Drobe B, de Saint-Andre R. The pre-myopic syndrome. Ophthalmic Physiol Opt. 1995;15:375-378.

18. Norton TT. Animal models of myopia: learning how vision controls the size of the eye. ILAR J. 1999;40:59-77.

19. Wildsoet CF. Active emmetropization: evidence for its existence and ramifications for clinical practice. Ophthalmic Physiol Opt. 1997;17:279-290.

20. Richler A, Bear JC. Refraction, nearwork, and education: a population study in Newfoundland. Acta Ophthalmol (Copenb). 1980; 58:468- 478 .

21. Zylbermann R, Landau D, Berson D. The influence of study habits on myopia in Jewish teenagers. J Pediatr Ophthalmol Strabismus. 1993;30:319-322.

22. Kinge B, Midelfart A, Jacobsen G, Rystad J. The influence of near-work on development of myopia among university students: a three-year longitudinal study among engineering students in Norway. Acta Ophthalmol Scand. 2000;78:26-29.

23. McBrien NA, Adams DW. A longitudinal investigation of adult-onset and adult-progression of myopia in an occupational group: refractive and biometric findings. Invest Ophthalmol Vis Sci. 1997;38:321-333.

24. Hepsen IF, Evereklioglu C, Bayramlar $\mathrm{H}$. The effect of reading and near-work on the development of myopia in emmetropic boys: a prospective, controlled, three-year follow-up study. Vision Res. 2001; $41: 2511-2520$.

25. Saw SM, Zhang MZ, Hong RZ, Fu ZF, Pang MH, Tan DT. Near work activity, night-lights, and myopia in the Singapore-China study. Arch Ophthalmol. 2002;120:620-627.

26. Mutti DO, Mitchell GL, Moeschberger ML, Jones LA, Zadnik K. Parental myopia, near work, school achievement, and children's refractive error. Invest Ophthalmol Vis Sci. 2002;43:3633-3640.

27. Haro C, Poulain I, Drobe B. Investigation of working distance in myopic and non-myopic children. Optom Vis Sci 2000;77: 189(suppl).

28. Parssinen O, Lyyra AL. Myopia and myopic progression among schoolchildren: a three-year follow-up study. Invest Ophthalmol Vis Sci. 1993;34:2794-2802.

29. Goss DA, Jackson TW. Clinical findings before the onset of myopia in youth: 3. Heterophoria. Optom Vis Sci. 1996;73:269-278.

30. Goss DA, Wolter KL. Nearpoint phoria changes associated with the cessation of childhood myopia progression. J Am Optom Assoc. 1999;70:764-768.

31. Gwiazda J, Grice K, Thorn F. Response AC/A ratios are elevated in myopic children. Ophthalmic Physiol Opt. 1999;19:173-179.

32. Jensen H. Myopia progression in young school children: a prospective study of myopia progression and the effect of a trial with 
bifocal lenses and beta blocker eye drops. Acta Ophthalmol Suppl. 1991;200:1-78.

33. Goss DA. Variables related to the rate of childhood myopia progression. Optom Vis Sci. 1990;67:631-636.

34. Manny RE, Hussein M, Scheiman M, Kurtz, D, Niemann K, Zinzer $\mathrm{K}$, COMET study group. Tropicamide (1\%): an effective cycloplegic agent for myopic children. Invest Ophthalmol Vis Sci. 2001; 42:1728-1735.

35. Zadnik K, Satariano WA, Mutti DO, Sholtz RI, Adams AJ. The effect of parental history of myopia on children's eye size. JAMA. 1994; 271:1323-1327.

36. Nelder JA. Regression, model-building and invariance in regression methods. J Roy Sta Soc Series A (General). 1968;131:303-329.

37. Little RC, Millken GA, Stroup WW, Wolfinger RD. SAS System for Mixed Models. Cary: SAS Institute Inc.; 1996.

38. Curtin BJ. The Myopias: Basic and Clinical Management. Philadelphia: Harper and Row; 1985:203-207.

39. Werner D, Press L. Clinical Pearls in Refractive Care. Boston: Butterworth-Heinemann; 2002:40.

40. Fulk GW, Cyert LA, Parker DE. A randomized trial of the effect of single-vision vs. bifocal lenses on myopia progression in children with esophoria. Optom Vis Sci. 2000;77:395-401.

41. Goss DA. Effect of bifocal lenses on the rate of childhood myopia progression. Am J Optom Physiol Opt. 1986;63:135-141.

42. Rosenfield M, Wong NN, Solan HA. Nearwork distances in children. Ophthalmic Physiol Opt. 2001;21:75-76.

43. Rah MJ, Mitchell GL, Bullimore MA, Mutti DO, Zadnik K. Prospective quantification of near work using the experience sampling method. Optom Vis Sci. 2001;78:496-502.

44. Smith EL III, Hung LF, Kee CS, Qiao Y. Effects of brief periods of unrestricted vision on the development of form-deprivation myopia in monkeys. Invest Ophthalmol Vis Sci. 2002;43:291-299.

45. Winawer J, Wallman J. Temporal constraints on lens compensation in chicks. Vision Res. 2002;42:2651-2668.

46. Mutti DO, Jones LA, Moeschberger ML, Zadnik K. AC/A ratio, age, and refractive error in children. Invest Ophthalmol Vis Sci. 2000; 41:2469-2478.

\section{APPENDIX}

The members of the COMET Study Group are as follows.

\section{Clinical Centers}

New England College of Optometry, Boston, Massachusetts: Daniel Kurtz (Principal Investigator); Erik Weissberg (Optometrist 6/99-present); Bruce Moore (Optometrist, until 6/99); Robert Owens (Primary Optician); Justin Smith (Clinic Coordinator 1/01-present); Sheila Martin (Clinic Coordinator, until 9/98); Joanne Bolden (Coordinator 10/98-present); Benny Jaramillo (Back-up Optician 3/00-present); Stacy Hamlett (Back-up Optician 6/98-5/00); Patricia Kowalski (Consulting Optometrist, until 6/01); Jennifer Hazelwood (Consulting Optometrist 7/01-present).

Pennsylvania College of Optometry, Philadelphia, Pennsylvania: Mitchell Scheiman (Principal Investigator); Kathleen Zinzer (Optometrist); Timothy Lancaster (Optician, until 6/99); Theresa Elliott (Optician, until 8/01); Mark Bernhardt (Optician 6/99-5/00); Dan Ferrara (Optician 7/00-7/01); Jeff Miles (Optician 8/01-present); Abby Grossman (Clinic Coordinator 8/01-present); Mariel Torres (Clinic Coordinator, until 6/00); Heather Jones (Clinic Coordinator 8/00-7/01); Melissa Madi-
gan-Carr (Coordinator 7/01-present); Theresa Sanogo (Back-up Coordinator 7/99-present); JoAnn Bailey (Consulting Optometrist).

University of Alabama at Birmingham School of Optometry, Birmingham, Alabama: Wendy Marsh-Tootle (Principal Investigator); Katherine Niemann (Optometrist 9/98-present); Kristine Becker (Ophthalmic Consultant 7/99-present); James Raley (Optician, until 4/99); Angela Rawden (Back-up Optician, until 9/98); Catherine Baldwin (Primary Optician \& Clinic Coordinator 10/98 -present); Nicholas Harris (Clinic Coordinator 3/98-9/99); Trana Mars (Back-up Clinic Coordinator 10/ 97-present); Robert Rutstein (Consulting Optometrist).

University of Houston College of Optometry, Houston, Texas: Ruth Manny (Principal Investigator); Connie Crossnoe (Optometrist); Sheila Deatherage (Optician); Charles Dudonis (Optician); Sally Henry (Clinic Coordinator, until 8/98); Jennifer McLeod (Clinic Coordinator 9/98 -present); Julio Quiralte (Backup Coordinator 1/98-present); Karen Fern (Consulting Optometrist).

\section{Resource Centers}

STUDY CHAIR'S OFFICE: New England College of Optometry, Boston, Massachusetts: Jane Gwiazda (Study Chair/Principal Investigator); Kenneth Grice (Study Coordinator, until 7/99); Christine Fortunato (Study Coordinator 8/99-9/00); Cara Weber (Study Coordinator 10/00 -present); Rosanna Pacella (Research Assistant, until 10/98); Thomas Norton (Consultant, University of Alabama at Birmingham).

COORDINATING CENTER: Department of Preventive Medicine, Stony Brook University Health Sciences Center, Stony Brook, New York: Leslie Hyman (Principal Investigator); M. Cristina Leske (Co-Principal Investigator); Mohamed Hussein (Co-Investigator/Biostatistician); Elinor Schoenfeld (Epidemiologist); Lynette Dias (Study Coordinator 6/98-present); Rachel Harrison (Study Coordinator 4/97-3/98); Jennifer Thomas (Assistant Study Coordinator 12/00 - present); Cristi Rau (Assistant Study Coordinator 2/99-11/00); Elissa Schnall (Assistant Study Coordinator 11/97-11/98); Wen Zhu (Senior Programmer); Ying Wang (Data Analyst 1/00-present); Ahmed Yassin (Data Analyst 1/98-1/99); Lauretta Passanant (Project Assistant 2/98-present); Maria Rodriguez (Project Assistant 10/00present); Allison Schmertz (Project Assistant 1/98 -12/98); Ann Park (Project Assistant 1/99-4/00); Phyllis Neuschwender (Administrative Assistant, until 11/99); Geeta Veeraraghavan (Administrative Assistant 12/99-4/01); Angela Santomarco (Administrative Assistant 7/01-present).

National Eye Institute, Bethesda, Maryland: Donald Everett (Program Director, Collaborative Clinical Trials Branch).

\section{Committees}

Data and Safety Monitoring Committee: Robert Hardy (Chair); Argye Hillis; Don Mutti; Richard Stone; Sr. Carol Taylor.

Executive Committee: Jane Gwiazda (Chair); Donald Everett; Leslie Hyman; Wendy Marsh-Tootle.

Steering Committee: Jane Gwiazda (Chair); Donald Everett; Mohamed Hussein; Leslie Hyman; M. Cristina Leske; Daniel Kurtz; Ruth Manny; Wendy Marsh-Tootle; Mitchell Scheiman; Thomas Norton. 